\title{
Plasma receptor for advanced glycation end products and clinical outcomes in acute lung injury
}

\author{
C S Calfee, ${ }^{1,2}$ L B Ware, ${ }^{3}$ M D Eisner,,${ }^{1,4}$ P E Parsons, ${ }^{5}$ B T Thompson,,${ }^{6,7}$ N Wickersham, ${ }^{3}$ \\ M A Matthay, ${ }^{1,2,4}$ the NHLBI ARDS Network
}

\section{See Editorial, p 1034}

- Supplementary acknowledgements are published online only at http://thorax.bmi.com/content/ vol63/issue12

${ }^{1}$ Department of Medicine Division of Pulmonary and Critical Care Medicine, University of California San Francisco, San Francisco, California, USA; ${ }^{2}$ Cardiovascular Research Institute, San Francisco, California, USA;

${ }^{3}$ Department of Medicine, Division of Allergy, Pulmonary and Critical Care, Vanderbilt University, Nashville, Tennessee, USA; ${ }^{4}$ Department of

Anesthesia, University of California San Francisco, San

Francisco, California, USA;

${ }^{5}$ Department of Medicine, Division of Pulmonary and Critical Care Medicine, University of Vermont Burlington, Vermont, USA; ${ }^{6}$ Department of Medicine Pulmonary and Critical Care Medicine Unit, Massachusetts General Hospital, Boston, Massachusetts, USA:

${ }^{7}$ Biostatistics Unit, Massachusetts General Hospital, Boston, Massachusetts, USA

Correspondence to: Dr C S Calfee, University of California, San Francisco, Pulmonary and Critical Care Division, 505 Parnassus Avenue, San Francisco, CA 94143-0130, USA; carolyn.calfee@ucsf.edu

Received 8 January 2008 Accepted 8 June 2008 Published Online First 19 June 2008

\begin{abstract}
Objectives: To determine whether baseline plasma levels of the receptor for advanced glycation end products (RAGE), a novel marker of alveolar type I cell injury, are associated with the severity and outcomes of acute lung injury, and whether plasma RAGE levels are affected by lower tidal volume ventilation.
\end{abstract}

Design, setting and participants: Measurement of plasma RAGE levels from 676 subjects enrolled in a large randomised controlled trial of lower tidal volume ventilation in acute lung injury.

Measurements and main results: Higher baseline plasma RAGE was associated with increased severity of lung injury. In addition, higher baseline RAGE was associated with increased mortality (OR for death 1.38 (95\% Cl 1.13 to 1.68$)$ per 1 log increment in RAGE; $p=0.002$ ) and fewer ventilator free and organ failure free days in patients randomised to higher tidal volumes. These associations persisted in multivariable models that adjusted for age, gender, severity of illness and the presence of sepsis or trauma. Plasma RAGE was not associated with outcomes in the lower tidal volume group ( $p=0.09$ for interaction in unadjusted analysis). In both tidal volume groups, plasma RAGE levels declined over the first 3 days; however, the decline was 15\% greater in the lower tidal volume group ( $p=0.02 ; 95 \% \mathrm{Cl} 2.4 \%$ to 25.0\%).

Conclusions: Baseline plasma RAGE levels are strongly associated with clinical outcomes in patients with acute lung injury ventilated with higher tidal volumes. Lower tidal volume ventilation may be beneficial in part by decreasing injury to the alveolar epithelium.

Acute lung injury (ALI) and its more severe form, the acute respiratory distress syndrome (ARDS), remain a major cause of acute respiratory failure and death in critically ill patients. ${ }^{1}$ Despite therapeutic advances in ventilation strategy ${ }^{2}$ and fluid management, ${ }^{3}$ mortality and morbidity remain high, and no effective pharmacological therapies for the syndrome have yet been identified. ${ }^{4}$ Increased understanding of the pathophysiology and pathogenesis of this syndrome, identification of predictors of poor outcomes and insight into the mechanisms of benefit of effective therapies such as low tidal volume ventilation may lead to further therapeutic advances.

The integrity of the alveolar-capillary barrier is critical to normal pulmonary function. Furthermore, impaired alveolar fluid clearance is known to be a central feature of the pathogenesis of ALI/ARDS. ${ }^{5} 6$ Type I epithelial cells cover $95 \%$ of the surface area of the alveolus ${ }^{7}$ and play a key role in epithelial integrity and alveolar fluid clearance. ${ }^{8-10}$ Given these important functions, the magnitude of damage to the alveolar type I cell could be a major determinant of the severity of ALI/ARDS and of its clinical outcomes. However, the search for a reliable, easily measured marker of alveolar type I cell injury in humans has proved difficult, and thus the importance of alveolar type I cell damage in humans with ALI remains unclear.

The receptor for advanced glycation end products (RAGE) was recently identified as a promising new marker of alveolar type I cell injury. ${ }^{11}$ RAGE is a member of the immunoglobulin superfamily that acts as a multi-ligand receptor and is involved in propagating inflammatory responses. ${ }^{12}{ }^{13}$ Immunoelectron microscopy has demonstrated that RAGE expression in the lung is primarily located on the basal surface of alveolar type I cells. ${ }^{14}{ }^{15}$ Although RAGE is produced by other cell types in other organs, such as vascular endothelium and nervous tissues, and is therefore not specific for alveolar type I cell injury, it is most abundant in the lung. ${ }^{13}$ In a murine model of ALI, elevated RAGE levels in bronchoalveolar lavage fluid and serum were directly related to the severity of experimentally induced lung injury. ${ }^{11}$ Similarly, in human subjects, plasma RAGE levels were significantly higher in patients with ALI than in patients with hydrostatic pulmonary oedema or normal controls. ${ }^{11}$ Elevated plasma RAGE levels have also been associated with an increased duration of mechanical ventilation and ICU length of stay in patients following lung transplantation. ${ }^{16}$ However, the potential pathogenic and prognostic value of RAGE has not been studied in patients with ALI.

The objective of this study was to determine whether plasma levels of RAGE are associated with the severity and clinical outcomes of ALI/ARDS in subjects enrolled in the ARDS Network's trial of a lower tidal volume, plateau pressure limited ventilation strategy. ${ }^{2}$ We hypothesised that increased baseline levels of RAGE would be associated with increased severity of ALI/ARDS and with poor clinical outcomes, including increased mortality and fewer ventilator free and organ failure free days. Furthermore, from a mechanistic perspective, we hypothesised that plasma RAGE levels measured at 3 days after enrolment would be preferentially decreased by a low tidal volume ventilation strategy.

\section{METHODS}

Clinical data and biological samples for this study were obtained from patients enrolled in a randomised controlled trial of lower tidal volume, plateau pressure limited ventilation conducted by 
the National Heart, Lung and Blood Institute's ARDS Network. ${ }^{2}$ This trial enrolled 861 patients to test the hypothesis that ventilation with a lower tidal volume, plateau pressure limited strategy would reduce mortality in patients with ALI. ${ }^{2}$ The details and results of the clinical trial have been previously published in full. ${ }^{2}$ Briefly, patients were randomised to ventilation with a tidal volume of either 6 or $12 \mathrm{ml} / \mathrm{kg}$ predicted body weight; once the benefit of the lower tidal volume strategy had been demonstrated, an additional 41 patients were assigned to the lower tidal volume to complete a factorialised trial of lisofylline versus placebo, ${ }^{17}$ and these patients are also included in this analysis. All patients were followed until death, 180 days or until discharge home with unassisted breathing. The trial demonstrated a significant reduction in 180 day mortality in the lower tidal volume treatment group ( $31 \%$ vs $40 \%, p=0.007$ ). In a factorial design, ketoconazole ${ }^{18}$ and lisofylline ${ }^{17}$ were also administered to subsets of patients as investigational therapies for ALI, neither of which affected mortality. Inclusion and exclusion criteria are described in the original publication. ${ }^{2}$ The institutional review boards of all involved hospitals approved the trial. ${ }^{2}$

\section{Assay procedures}

We measured baseline (day 0, pre-randomisation) and day 3 plasma levels of RAGE using a commercially available enzyme linked immunoassay (Human RAGE Quantikine ELISA; R\&D Systems, Minneapolis, Minnesota, USA) in 676 patients from the original clinical trial. RAGE levels were not measured in all 902 patients because of limited plasma availability.

\section{Statistical methods}

Statistical analysis was performed with STATA/SE 9.2 (College Station, Texas, USA). To compare baseline levels of RAGE in two group comparisons, we used Mann-Whitney rank sum tests; for multiple group comparisons, we used the KruskalWallis test. Because RAGE levels were not normally distributed,

Table 1 Baseline characteristics of the study sample

\begin{tabular}{lccl}
\hline Characteristic & $\begin{array}{c}\text { RAGE measured } \\
(\mathbf{n}=\mathbf{6 7 6})\end{array}$ & $\begin{array}{c}\text { Not measured } \\
(\mathbf{n}=\mathbf{2 2 6})\end{array}$ & $\mathbf{p}$ Value \\
\hline Age (years) (mean (SD)) & $51(17)$ & $52(18)$ & 0.43 \\
Female gender & $394(58)$ & $143(63)$ & 0.19 \\
Race/ethnicity & & & 0.61 \\
$\quad$ White & $497(69)$ & $164(73)$ & \\
$\quad$ Black & $118(17)$ & $37(16)$ & \\
$\quad$ Hispanic & $39(6)$ & $12(5)$ & \\
$\quad$ Asian/Pacific Islander & $17(3)$ & $9(4)$ & \\
$\quad$ Native American & $3(0.4)$ & $3(1.3)$ & \\
$\quad$ Other & $2(0.3)$ & $1(0.4)$ & \\
APACHE III score (mean (SD)) & $82.0(27.5)$ & $86.5(31.5)$ & 0.05 \\
Sepsis as primary ALI risk factor & $173(26)$ & $63(28)$ & 0.50 \\
Trauma as primary ALI risk factor & $63(9)$ & $33(15)$ & 0.03 \\
Pa0 $2:$ Fio 2 ratio (mean (SD)) & $128(58)$ & $135(58)$ & 0.11 \\
Diabetes & $97(14)$ & $29(13)$ & 0.55 \\
End stage renal disease & $18(3)$ & $2(1)$ & 0.12 \\
Lower tidal volume arm & $352(52)$ & $121(54)$ & 0.70 \\
WBC (/10 ${ }^{3}$ ) (mean (SD)) & $14.9(1.0)$ & $14.5(1.1)$ & 0.55 \\
Lowest MAP (mean (SD)) & $62.1(14)$ & $61.5(14)$ & 0.57 \\
\hline
\end{tabular}

Data reported as $\mathrm{n}(\%)$ unless otherwise specified.

ALI, acute lung injury; APACHE, Acute Physiology and Chronic Health Evaluation; $\mathrm{MAP}$, mean arterial pressure; $\mathrm{Fi}_{2}$, fractional inspired oxygen; $\mathrm{PaO}_{2}$, arterial oxygen tension; RAGE, receptor for advanced glycation end products; WBC, white blood cell count. we applied natural log transformation to the plasma RAGE levels in order to apply linear models. We first tested the impact of RAGE on mortality in an unadjusted logistic regression and then created a multivariable logistic model to control for baseline clinical features that may have affected outcomes, including age, gender, sepsis or trauma as aetiology of lung injury, and severity of illness, as reflected by Acute Physiology and Chronic Health Evaluation (APACHE) III score. ${ }^{19}$ These covariates were selected on the basis of the bivariate analysis results (all were associated with mortality, with $\mathrm{p}<0.05$, with the exception of gender, which was associated with baseline RAGE) and prior clinical research about predictors of mortality in ALI. ${ }^{20}$ We performed similar analyses using linear regression models for the outcomes of ventilator free days and organ failure free days. In addition, in a pre-specified analysis, plasma RAGE levels were divided into quartiles to test for a non-linear relationship with outcomes in similar regressions. All analyses were stratified by ventilation strategy because of an observed interaction between baseline plasma RAGE level and ventilation strategy; this interaction was the only interaction tested and was initially sought after a difference in the odds ratios for death stratified by ventilation strategy was observed. Unstratified analyses are presented in the online supplement. A $p$ value of $<0.20$ was considered potentially important for interactions $^{21}$; for all other analyses, a $p$ value of $<0.05$ was considered significant. Logistic models were checked using the Hosmer-Lemeshow test and the link test ${ }^{22}$; linear models were evaluated using residual based diagnostics. Analysis of covariance was used to test the impact of lower tidal volume ventilation on the change in RAGE levels from day 0 to day 3.

\section{RESULTS}

\section{Baseline clinical characteristics of subjects}

Baseline plasma samples were available for RAGE measurements in 676 of the 902 original study subjects. The clinical characteristics of subjects who had plasma samples available were similar to those who did not have plasma samples available (table 1), with the exception of a lower incidence of trauma as the aetiology of ALI and a slightly lower mean APACHE III score. Baseline plasma RAGE levels did not differ by clinical risk factor for ALI ( $p=0.54$ ) or by race/ethnicity but did differ by gender (median in men $5536 \mathrm{pg} / \mathrm{ml}$ vs $3360 \mathrm{pg} / \mathrm{ml}$ in women; $\mathrm{p}<0.0001$ ). Plasma RAGE levels did not differ based on the presence of end stage renal disease, diabetes, cirrhosis of the liver, a history of malignancy or immunosuppression, or a history of the acquired immunodeficiency syndrome.

\section{Severity of ALI/ARDS}

Higher baseline levels of plasma RAGE were significantly associated with more severe ALI/ARDS, as reflected by measures of pulmonary physiology, including lower fractional inspired oxygen/arterial oxygen tension $\left(\mathrm{PaO}_{2} / \mathrm{FiO}_{2}\right)$ ratio, lower compliance, and higher clinical and radiographic lung injury scores (figs 1 and 2). ${ }^{23}$ Likewise, higher baseline plasma RAGE levels were associated with higher pre-randomisation tidal volume $(r=0.12 ; p=0.002)$ and plateau pressure $(r=0.22$; $p<0.0001)$. Higher baseline levels of plasma RAGE were also associated with higher APACHE scores $(r=0.17$; $<<0.0001)$.

\section{Clinical outcomes}

In unadjusted analyses, baseline plasma RAGE was strongly associated with poor clinical outcomes. Baseline RAGE was significantly higher in subjects who died compared with those 


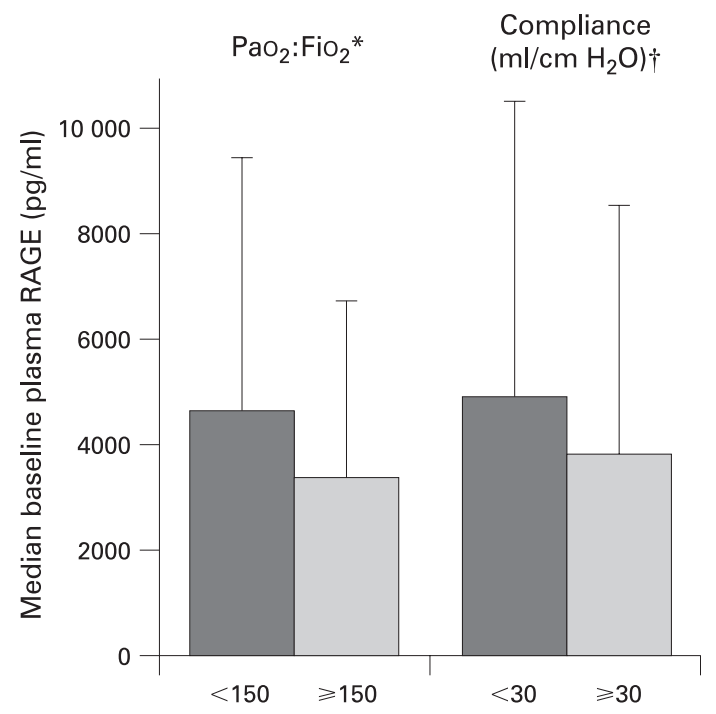

Figure 1 Higher baseline plasma receptor for advanced glycation end products (RAGE) was associated with more severe disturbances in pulmonary physiology ( $\mathrm{n}=676$ for fractional inspired oxygen/arterial oxygen tension $\left(\mathrm{PaO}_{2} / \mathrm{FiO}_{2}\right) ; \mathrm{n}=522$ for compliance). Median and interquartile range are shown. ${ }^{*} p=0.008 ; \dagger p=0.01$

who survived (median (IQR) in non-survivors 5327 (2250 to $10112) \mathrm{pg} / \mathrm{ml}$ vs in survivors 3618 (1916 to 7402$) \mathrm{pg} / \mathrm{ml}$; $p=0.001)$. However, on further analysis of the association between baseline plasma RAGE and death, an interaction between baseline plasma RAGE and ventilation strategy was detected $(p=0.09)$. When the analysis was stratified by ventilation strategy, baseline RAGE was predictive of death only in the group randomised to the $12 \mathrm{ml} / \mathrm{kg}$ strategy (fig 3, table 2) (odds ratio (OR) per 1 log increment in RAGE 1.38; 95\% confidence interval (CI) 1.13 to 1.68; $p=0.002$ ). Baseline RAGE was not predictive of death in the lower tidal volume group (OR 1.07; $95 \%$ CI 0.86 to $1.32 ; p=0.53$ ).

Baseline plasma RAGE was also strongly associated with increased morbidity from ALI in the $12 \mathrm{ml} / \mathrm{kg}$ ventilation group (table 3). Specifically, for every 1 log increase in plasma RAGE, ventilator free days decreased by 1.57 (95\% CI -2.57 to -0.56 days; $p=0.002$ ) and organ failure free days decreased by 2.44 ( $95 \%$ CI -3.44 to -1.44 days, $p<0.001)$. Baseline plasma RAGE was not associated with increased morbidity in the $6 \mathrm{ml} / \mathrm{kg}$ group (table 3 ).

\section{Multivariable models}

The strong association between baseline plasma RAGE and poor clinical outcomes in the $12 \mathrm{ml} / \mathrm{kg}$ group persisted in multivariable models. After adjusting for age, gender, APACHE III score and the presence of sepsis or trauma as a risk factor for ALI, higher baseline plasma RAGE remained strongly associated with an increased odds of death at 180 days in the $12 \mathrm{ml} / \mathrm{kg}$ group (table 2). Additional adjustments for pre-existing diabetes and end stage renal disease had no appreciable impact on the results of these regressions; similarly, including all precipitating risk factors for ALI in the model had no appreciable impact on the results. As with the mortality analyses, higher baseline plasma RAGE remained associated with a lower number of ventilator free and organ failure free days in multivariable linear models in the $12 \mathrm{ml} / \mathrm{kg}$ group, adjusting for the same clinical covariates (table 3). Multivariable models revealed no

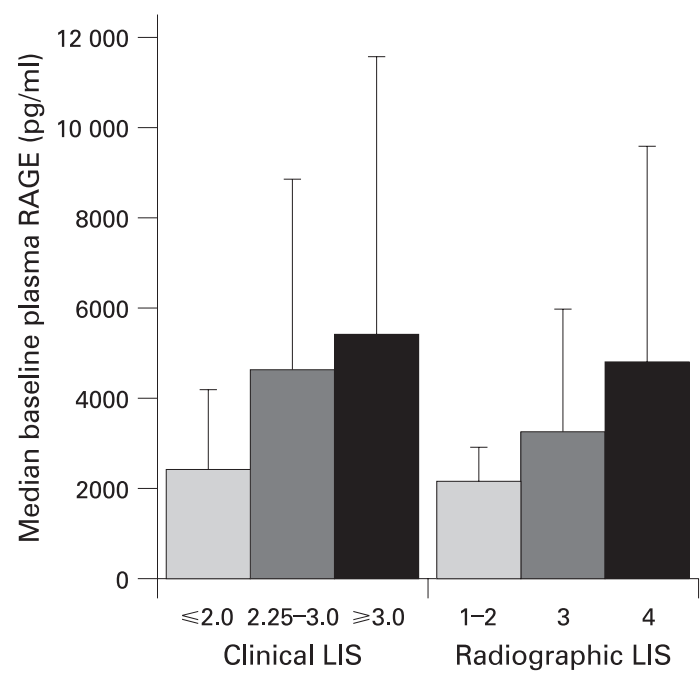

Figure 2 Increasing clinical and radiographic lung injury scores (LIS), reflecting increasing severity of lung injury, ${ }^{23}$ were associated with increasing baseline plasma receptor for advanced glycation end products (RAGE) ( $n=669$ ). Median and interquartile range are shown. For clinical LIS: Kruskal-Wallis $p=0.0008$; test for trend $p<0.001$. For radiographic LIS: Kruskal-Wallis $p=0.001$; test for trend $p<0.001$.

association between baseline RAGE and clinical outcomes in the $6 \mathrm{ml} / \mathrm{kg}$ group (table 3 ).

To further study the relationship of baseline plasma RAGE to clinical outcomes, the multivariable analyses were repeated using quartiles of RAGE, again controlling for the same clinical covariates (age, gender, APACHE III score, sepsis and trauma) and stratifying by ventilation strategy. In the $12 \mathrm{ml} / \mathrm{kg}$ group, this analysis demonstrated an increased risk of death for the highest quartile of RAGE compared with the lowest quartile (OR 2.73, 95\% CI 1.31 to 5.68 ; overall $p=0.003$ ) (table 4). In the $6 \mathrm{ml} / \mathrm{kg}$ group, RAGE by quartile was not significantly associated with mortality (data not shown). Linear regression

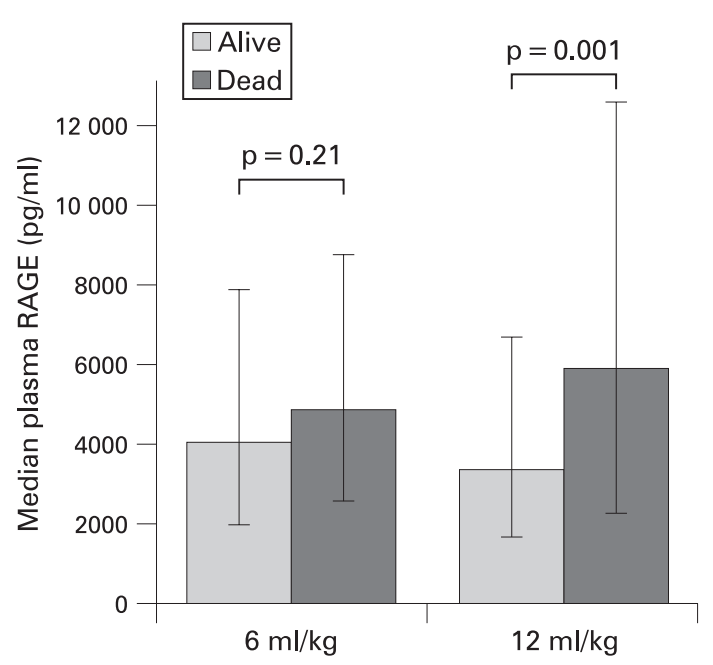

Figure 3 Baseline plasma receptor for advanced glycation end products (RAGE) by survival status, stratified by ventilation strategy $(n=676)$. Baseline plasma RAGE was associated with death at 180 days only in the $12 \mathrm{ml} / \mathrm{kg}$ group. Median (interquartile range) plasma RAGE in each group is shown. Comparisons were made using the Mann-Whitney (Wilcoxon) rank sum test. 
Table 2 Baseline plasma RAGE and death at 180 days among patients with ALI/ARDS, stratified by ventilator strategy

\begin{tabular}{llll}
\hline Model & $\begin{array}{l}\text { Tidal volume } \\
\text { group }(\mathbf{m l} / \mathbf{k g})\end{array}$ & $\begin{array}{l}\text { OR for death at } \\
\mathbf{1 8 0} \text { days }(\mathbf{9 5 \%} \mathbf{~ C l})\end{array}$ & p Value \\
\hline $\begin{array}{l}\text { Baseline plasma RAGE }(1 \text { log increment), } \\
\text { unadjusted model }\end{array}$ & 12 & $1.38(1.13$ to 1.68$)$ & 0.002 \\
$\begin{array}{l}\text { Baseline plasma RAGE }(1 \log \text { increment), } \\
\text { multivariable model* }\end{array}$ & 12 & $1.41(1.12$ to 1.78$)$ & 0.004 \\
$\begin{array}{l}\text { Baseline plasma RAGE }(1 \text { log increment), } \\
\text { unadjusted model }\end{array}$ & 6 & $1.07(0.86$ to 1.32$)$ & 0.53 \\
$\begin{array}{l}\text { Baseline plasma RAGE }(1 \text { log increment), } \\
\text { multivariable model* }\end{array}$ & 6 & $1.03(0.81$ to 1.31$)$ & 0.80
\end{tabular}

Multivariable model adjusts for age, gender, APACHE III score and presence of sepsis or trauma as ALI risk factors. In the $12 \mathrm{ml} / \mathrm{kg}$ model: Hosmer-Lemeshow, $p=0.24$; link test hatsq $=0.22 .{ }^{22}$ In the $6 \mathrm{ml} / \mathrm{kg}$ model: Hosmer-Lemeshow, $p=0.35$; link test hatsq $=0.99$. All transformations use natural logarithms.

${ }^{*} p$ Value for interaction between baseline RAGE and death in multivariable model is 0.06 .

ALI, acute lung injury; APACHE, Acute Physiology and Chronic Health Evaluation; ARDS, acute respiratory distress syndrome; RAGE, receptor for advanced glycation end products.

models for ventilator free days and organ failure free days using quartiles of RAGE revealed similar findings (table 4).

\section{Impact of lower tidal volume strategy}

The impact of the lower tidal volume ventilation strategy on the change in RAGE levels over the first 3 days of the study was measured using analysis of covariance. There were 599 patients with paired day 0 and day 3 samples available for analysis. In both the $6 \mathrm{ml} / \mathrm{kg}$ and $12 \mathrm{ml} / \mathrm{kg}$ groups, plasma RAGE levels declined over the first 3 days; however, there was a $15 \%$ greater decline in the $6 \mathrm{ml} / \mathrm{kg}$ group compared with the $12 \mathrm{ml} / \mathrm{kg}$ group ( $p=0.02 ; 95 \%$ CI $2.4 \%$ to $25.0 \%$ ) (fig 4 ).

Given the observed interaction between baseline plasma RAGE and ventilator strategy, we compared the effect of low tidal volume ventilation on mortality in the highest compared with the lowest quartile of baseline RAGE. In the highest quartile of baseline RAGE, the OR for death in subjects treated with lower tidal volume ventilation was 0.36 compared with those treated with higher tidal volumes (95\% CI 0.19 to 0.67 ; $p=0.001)$. In comparison, in the lowest quartile of baseline RAGE, the OR for death in subjects treated with lower tidal volumes was 0.52 ( $95 \%$ CI 0.26 to $1.00 ; p=0.05$ ).

\section{DISCUSSION}

In a large, multicentre, randomised, controlled trial of ventilation strategy in ALI, higher baseline plasma RAGE levels were associated with worse clinical outcomes in patients randomised to higher tidal volumes, including increased mortality and fewer ventilator free and organ failure free days. These associations persisted even after adjusting for other important determinants of outcome, including age, aetiology of ALI and severity of illness. In patients randomised to lower tidal volumes, plasma RAGE was not predictive of outcomes. Plasma RAGE was associated with measures of severity of lung injury, including $\mathrm{PaO}_{2}: \mathrm{FiO}_{2}$, quasi-static compliance and lung injury score. Furthermore, lower tidal volume ventilation amplified the decline in RAGE levels over the first 3 days of the study, suggesting that ventilation with a $6 \mathrm{ml} / \mathrm{kg}$ strategy may cause less injury to alveolar type I cells than ventilation with higher tidal volumes.

Although RAGE is not specific for alveolar epithelial injury, several findings in this study and in prior work provide supportive evidence that the primary source of plasma RAGE in our patients is the alveolar type I cell. In our previous work on RAGE in animal models of ALI, both acid aspiration and lipopolysaccharide induced lung injury resulted in RAGE release into the bronchoalveolar lavage fluid at much higher levels than in plasma, suggesting a pulmonary source for plasma RAGE in the setting of ALI. ${ }^{11}$ A similar pattern was found in human oedema fluid and plasma samples from subjects with ALI. ${ }^{11}$ Likewise, in a recently published isolated perfused human lung preparation in which the lung is the only possible source of RAGE, perfusate levels of RAGE were significantly and inversely associated with alveolar fluid clearance, suggesting that RAGE

Table 3 Baseline plasma RAGE and other clinical outcomes at 180 days among patients with ALI/ARDS, stratified by ventilation strategy

\begin{tabular}{llllll}
\hline $\begin{array}{l}\text { Ventilator } \\
\text { group }\end{array}$ & Model & $\begin{array}{l}\text { Mean difference in } \\
\text { ventilator free days* } \\
(\mathbf{9 5 \%} \mathbf{~ C l})\end{array}$ & p Value & $\begin{array}{l}\text { Mean difference in } \\
\text { organ failure free } \\
\text { days } \dagger \text { (95\% Cl) }\end{array}$ & p Value \\
\hline $12 \mathrm{ml} / \mathrm{kg}$ & $\begin{array}{l}\text { Baseline plasma RAGE }(1 \mathrm{log} \\
\text { increment), unadjusted model }\end{array}$ & $-1.57(-2.57$ to -0.56$)$ & 0.002 & $-2.44(-3.44$ to -1.44$)$ & $<0.001$ \\
\hline $\begin{array}{l}\text { Baseline plasma RAGE (1 log } \\
\text { increment), multivariable model }\end{array}$ & $-1.15(-2.10$ to -0.20$)$ & 0.02 & $-1.98(-2.94$ to -1.03$)$ & $<0.001$ \\
\hline $\mathrm{ml} / \mathrm{kg}$ & $\begin{array}{l}\text { Baseline plasma RAGE (1 log } \\
\text { increment), unadjusted model } \\
\text { Baseline plasma RAGE (1 log } \\
\text { increment), multivariable model }\end{array}$ & $-0.57(-1.59$ to 0.45$)$ & 0.27 & $-1.01(-2.06$ to 0.03$)$ & 0.06 \\
\hline
\end{tabular}

Multivariable model adjusts for age, gender, APACHE III score, and presence of sepsis or trauma as ALI risk factors. Model checking performed using residual based diagnostics. All transformations use natural logarithms.

${ }^{*} \mathrm{p}$ Value for interaction between ventilator strategy and baseline RAGE in univariate model is 0.17 ; in multivariate model, $\mathrm{p}=0.12$.

$\dagger p$ value for interaction between ventilator strategy and baseline RAGE in univariate model is 0.05 ; in multivariate model, $p=0.04$.

ALI, acute lung injury; APACHE, Acute Physiology and Chronic Health Evaluation; ARDS, acute respiratory distress syndrome;

RAGE, receptor for advanced glycation end products. 
Table 4 Quartiles of RAGE and clinical outcomes in the $12 \mathrm{ml} / \mathrm{kg}$ group

\begin{tabular}{|c|c|c|c|c|}
\hline Outcome & Predictor & $\begin{array}{l}\text { Mean effect, } \\
\text { compared with first } \\
\text { quartile }(95 \% \mathrm{CI})\end{array}$ & $\begin{array}{l}\text { p Value for } \\
\text { predictor }\end{array}$ & $\begin{array}{l}p \text { Value for } \\
\text { trend }\end{array}$ \\
\hline \multirow[t]{3}{*}{ Odds ratio for death at 180 days } & Second quartile RAGE & $0.77(0.37$ to 1.57$)$ & 0.003 & 0.008 \\
\hline & Third quartile RAGE & $0.86(0.41$ to 1.80$)$ & & \\
\hline & Fourth quartile RAGE & $2.73(1.31$ to 5.68$)$ & & \\
\hline \multirow[t]{3}{*}{ Ventilator free days } & Second quartile RAGE & $0.1(-3.0$ to 3.1$)$ & & \\
\hline & Third quartile RAGE & $0.1(-3.0$ to 3.4$)$ & 0.01 & 0.01 \\
\hline & Fourth quartile RAGE & $-4.2(-7.3$ to -1.1$)$ & & \\
\hline \multirow[t]{3}{*}{ Organ failure free days } & Second quartile RAGE & $-2.4(-5.5$ to 0.8$)$ & & \\
\hline & Third quartile RAGE & $-2.3(-5.6$ to 1.0$)$ & 0.001 & 0.0003 \\
\hline & Fourth quartile RAGE & $-6.4(-9.5$ to -3.2$)$ & & \\
\hline
\end{tabular}

All analyses adjusted for age, gender, APACHE III, sepsis and trauma.

APACHE, Acute Physiology and Chronic Health Evaluation; RAGE, receptor for advanced glycation end products.

quantitatively reflects alveolar epithelial damage. ${ }^{24}$ In the current study, the finding that plasma RAGE levels were associated with the severity of pulmonary physiological disturbances $\left(\mathrm{PaO}_{2} / \mathrm{FiO}_{2}\right.$ ratio, compliance and plateau pressure) also supports an alveolar epithelial source of RAGE.

Despite this evidence, it is important to emphasise that nonpulmonary sources of RAGE may contribute to plasma levels in the setting of ALI, including release from non-pulmonary large vessel endothelial surfaces and systemic inflammation. Moreover, plasma RAGE levels may have been affected by the permeability of the alveolar-capillary interface, although this phenomenon would if anything strengthen its utility as a marker of lung injury. While the precise function of RAGE remains unclear, evidence is mounting that RAGE plays an important role in regulating inflammation. The primary ligands for RAGE are advanced glycation end products (AGE), nonenzymatically glycated adducts of endogenous proteins, lipids and nucleic acids whose formation is increased in the presence of hyperglycaemia or oxidant stress. ${ }^{12}$ In addition to AGE, RAGE has several alternative ligands, including proinflammatory molecules such as S100 and high mobility group box $1 .^{12}$ RAGE-ligand interaction mediates nuclear factor $\kappa \mathrm{B}$ activation and seems thereby to play a role in propagating and sustaining
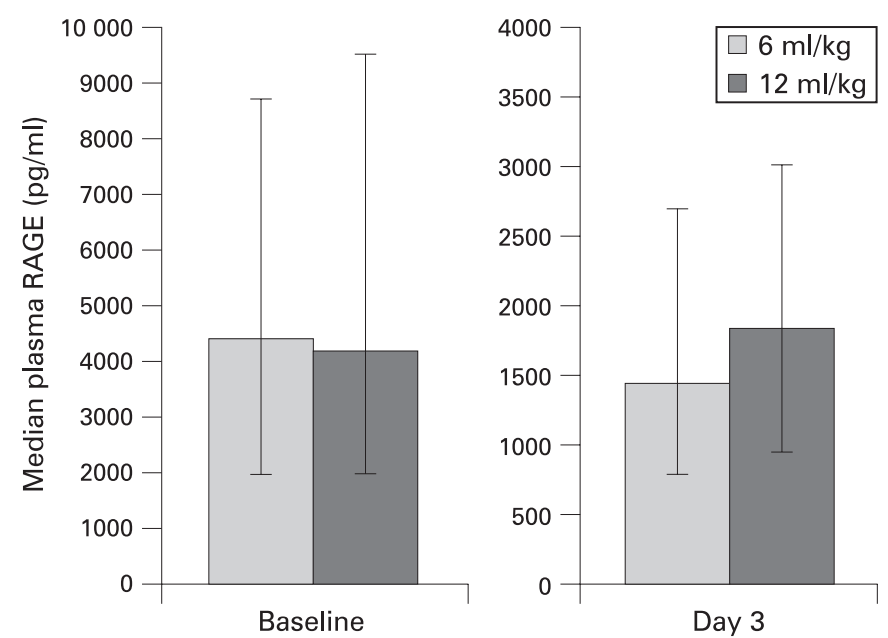

Figure 4 Plasma levels of receptor for advanced glycation end products (RAGE) declined in both the $6 \mathrm{ml} / \mathrm{kg}$ and $12 \mathrm{ml} / \mathrm{kg}$ groups over the first 3 days of the study $(n=599)$; however, the decline was $14.6 \%$ greater in the lower tidal volume group $(\mathrm{p}=0.02 ; 95 \% \mathrm{Cl} 2.4 \%$ to $25.0 \%$ ). Note the $y$ axis scale differs between the two sides of the figure. systemic inflammatory responses. ${ }^{25} 26$ Thus although we set out to study RAGE as a marker of alveolar epithelial injury, it is possible that the association between plasma RAGE and clinical outcomes of ALI observed in our study derives in part from the role of RAGE in an inflammatory cascade rather than purely from its release from alveolar type I cells. If plasma RAGE levels measured in our study primarily reflect the role of RAGE in inflammation, then the interaction between RAGE and ventilator strategy lends further credence to the hypothesis that low tidal volume ventilation decreases systemic inflammation. $^{27} 28$

The finding of effect modification in this study - that is, that the predictive value of RAGE differed by tidal volume strategywas particularly informative. If plasma RAGE primarily reflects alveolar epithelial injury, this interaction implies that patients with more severe alveolar epithelial injury are most susceptible to the additional ventilator induced lung injury that may be generated by higher tidal volumes. This interpretation is reinforced by the finding that lower tidal volume ventilation preferentially amplified the decline in RAGE levels over the first 3 days of the study. Lower tidal volume ventilation preferentially affects the plasma levels of several biomarkers that have prognostic value in ALI/ARDS, including markers of inflammation (interleukins 6 and 8), ${ }^{27}$ alveolar type II cell injury (surfactant protein D) ${ }^{29}$ and soluble tumour necrosis factor receptor 1,30 which may reflect neutrophil-endothelial interaction and/or epithelial injury. When taken in concert with the finding of effect modification by lower tidal volume ventilation, the amplified decline in RAGE levels over time in patients in the $6 \mathrm{ml} / \mathrm{kg}$ group provides important evidence that a lung protective ventilation strategy is effective at least in part by decreasing injury to the alveolar epithelium.

The results of this study also suggest that the degree of injury to the alveolar type I cell is an important determinant of outcomes in human ALI. Type I cells cover the vast majority of the alveolar surface and form a key component of the alveolar capillary barrier. In addition to this role, type I cells have been traditionally thought to be primarily responsible for gas exchange and osmotically driven water transport ${ }^{10}$ whereas type II cells have been thought to be primarily responsible for surfactant production, ion transport and alveolar fluid clearance. ${ }^{31}$ Recent research has suggested, however, that type I cells play a key role in ion and vectorial fluid transport as well, making their function all the more critically relevant to the pathogenesis and resolution of lung injury, ${ }^{92}$ since impaired alveolar fluid clearance is clearly linked to poor clinical outcomes in ALI. ${ }^{5}{ }^{6}$ The specific association between alveolar type I cell damage and the severity of lung injury has been 
demonstrated in rat models of ALI, ${ }^{11}{ }^{33}$ but the lack of a human marker of type I cell injury has precluded quantitative testing of this relationship (and the relationship of type I cell injury to clinical outcomes) in human studies until now.

The finding that plasma RAGE levels differed at baseline by gender was surprising and has not to our knowledge been reported in other clinical settings. Men on average have larger lungs than women, and thus by definition should have more alveolar epithelial cells; however, the magnitude of difference detected in this sample seems unlikely to be due to differences in lung mass. Although gender has not been associated with outcomes in most studies of ALI, we included gender as a covariate in all of our multivariable models to account for any confounding that might have occurred because of this difference in baseline plasma RAGE levels.

This study has some limitations. Firstly, plasma RAGE was not measured in all participants in the original trial, but only in subjects for whom plasma samples were still available. Although our analyses revealed few differences between subjects who had plasma available and those who did not, it remains possible that systematic factors contributed in some way to plasma availability. Secondly, the selection of potential confounders for the multivariable models was limited to clinical data collected by the original ARDS Network studies. Several chronic diseases that may affect clinical outcomes may also impact on RAGE levels; for instance, subjects with diabetes mellitus $^{34}$ and end stage renal disease ${ }^{35}$ have been shown to have elevated levels of plasma RAGE, whereas subjects with coronary artery disease, ${ }^{36}$ rheumatoid arthritis, ${ }^{37}$ Alzheimer's disease ${ }^{38}$ and essential hypertension ${ }^{39}$ have been shown to have decreased levels of plasma RAGE. Although we were able to control for diabetes mellitus and pre-existing dialysis dependent renal failure in sensitivity analyses (and demonstrate that they had no impact on our results), coronary artery disease, rheumatoid arthritis, Alzheimer's disease and hypertension were not recorded in the original dataset. Thus it is possible that chronic diseases present in some study subjects are unmeasured confounders in our analyses. Thirdly, the finding of an interaction between baseline RAGE and ventilation strategy should, like all subgroup analyses, be interpreted with caution, particularly as the criteria used to determine the potential importance of the interaction were less stringent than for other analyses. The use of a less stringent $p$ value for interaction analyses is common in the epidemiological literature ${ }^{21} 40$ although the optimum cut-off remains controversial. ${ }^{40}$ Moreover, when the data are analysed without stratification to account for the interaction, baseline RAGE is significantly predictive of death and the other key clinical outcomes in the cohort as a whole in both unadjusted and multivariable models (data available in online supplement). Finally, plasma samples used in this study had been stored for several years at $-70^{\circ} \mathrm{C}$; whether this extended storage has any effects on plasma biomarker levels remains unknown.

In conclusion, in this analysis of data from a large randomised controlled trial, baseline levels of plasma RAGE were independently associated with clinical outcomes in ALI/ARDS in patients randomised to traditional higher tidal volumes. In this group, higher baseline plasma RAGE levels were associated with increased mortality and fewer ventilator free and organ failure free days. In addition, lower tidal volume ventilation amplified the decline in RAGE levels over time. These findings provide new evidence that lower tidal volume ventilation may be beneficial at least in part by decreasing injury to the alveolar epithelium.
Acknowledgements: Details of acknowledgements are in the supplementary material online.

Funding: This work was supported by contracts (N01-HR 46054, 46055, 46056, 46057, 46058, 46059, 46060, 46061, 46062, 46063, and 46064) from the National Heart, Lung, and Blood Institute (NHLBI). MAM was supported by NHLBI Grant HL 51856. CSC was supported by KL2RR024130 from the National Center for Research Resources, a component of the NIH, and by the Flight Attendant Medical Research Institute. LBW was supported by NHLBI Grant HL081332.

Competing interests: None.

Ethics approval: Ethics approval was obtained

\section{REFERENCES}

1. Rubenfeld GD, Caldwell E, Peabody E, et al. Incidence and outcomes of acute lung injury. N Engl J Med 2005;353:1685-93.

2. The Acute Respiratory Distress Syndrome Network. Ventilation with lower tidal volumes as compared with traditional tidal volumes for acute lung injury and the acute respiratory distress syndrome. N Engl J Med 2000;342:1301-8.

3. The Acute Respiratory Distress Syndrome Network. Comparison of two fluidmanagement strategies in acute lung injury. N Engl J Med 2006;354:2564-75.

4. Cepkova M, Matthay MA. Pharmacotherapy of acute lung injury and the acute respiratory distress syndrome. J Intensive Care Med 2006;21:119-43.

5. Ware LB, Matthay MA. Alveolar fluid clearance is impaired in the majority of patients with acute lung injury and the acute respiratory distress syndrome. Am J Respir Crit Care Med 2001:163:1376-83.

6. Matthay MA, Wiener-Kronish JP. Intact epithelial barrier function is critical for the resolution of alveolar edema in humans. Am Rev Respir Dis 1990;142:1250-7.

7. Stone KC, Mercer RR, Freeman BA, et al. Distribution of lung cell numbers and volumes between alveolar and nonalveolar tissue. Am Rev Respir Dis 1992;146:454-6.

8. Johnson MD, Bao HF, Helms MN, et al. Functional ion channels in pulmonary alveolar type I cells support a role for type I cells in lung ion transport. Proc Natl Acad Sci U S A 2006;103:4964-9.

9. Johnson MD, Widdicombe JH, Allen L, et al. Alveolar epithelial type I cells contain transport proteins and transport sodium, supporting an active role for type I cells in regulation of lung liquid homeostasis. Proc Natl Acad Sci U S A 2002;99:1966-71.

10. Ma T, Fukuda N, Song $Y$, et al. Lung fluid transport in aquaporin-5 knockout mice. $J$ Clin Invest 2000;105:93-100.

11. Uchida T, Shirasawa M, Ware LB, et al. Receptor for advanced glycation endproducts is a marker of type I cell injury in acute lung injury. Am J Respir Crit Care Med 2006;173:1008-15

12. Bierhaus A, Humpert PM, Morcos M, et al. Understanding RAGE, the receptor for advanced glycation end products. J Mol Med 2005;83:876-86.

13. Schmidt AM, Yan SD, Yan SF, et al. The multiligand receptor RAGE as a progression factor amplifying immune and inflammatory responses. J Clin Invest 2001;108:949-55.

14. Fehrenbach $\mathbf{H}$, Kasper M, Tschernig T, et al. Receptor for advanced glycation endproducts (RAGE) exhibits highly differential cellular and subcellular localisation in rat and human lung. Cell Mol Biol (Noisy-Le-Grand) 1998;44:1147-57.

15. Shirasawa M, Fujiwara N, Hirabayashi S, et al. Receptor for advanced glycation end-products is a marker of type I lung alveolar cells. Genes Cells 2004;9:165-74.

16. Calfee CS, Budev MM, Matthay MA, et al. Plasma receptor for advanced glycation end-products predicts duration of ICU stay and mechanical ventilation in patients after lung transplantation. J Heart Lung Transplant 2007;26:675-80.

17. The Acute Respiratory Distress Syndrome Network. Randomized, placebocontrolled trial of lisofylline for early treatment of acute lung injury and acute respiratory distress syndrome. Crit Care Med 2002;30:1-6.

18. The Acute Respiratory Distress Syndrome Network. Ketoconazole for early treatment of acute lung injury and acute respiratory distress syndrome: a randomized controlled trial. JAMA 2000;283:1995-2002.

19. Knaus WA, Wagner DP, Draper EA, et al. The APACHE III prognostic system. Risk prediction of hospital mortality for critically ill hospitalized adults. Chest 1991;100:1619-36.

20. Eisner MD, Thompson T, Hudson LD, et al. Efficacy of low tidal volume ventilation in patients with different clinical risk factors for acute lung injury and the acute respiratory distress syndrome. Am J Respir Crit Care Med 2001;164:231-6.

21. Jewell N. Statistics for epidemiology. London: Chapman and Hall, 2004.

22. Vittinghoff E, Glidden D, Shiboski SC, et al. Regression methods in biostatistics: linear, logistic, survival, and repeated measures models. New York: Springer Science and Business Media; 2005.

23. Murray JF, Matthay MA, Luce JM, et al. An expanded definition of the adult respiratory distress syndrome. Am Rev Respir Dis 1988;138:720-3.

24. Frank JA, Briot R, Lee JW, et al. Physiological and biochemical markers of alveolar epithelial barrier dysfunction in perfused human lungs. Am J Physiol Lung Cell Mol Physiol 2007;293:L52-9

25. Liliensiek B, Weigand Ma, Bierhaus A, et al. Receptor for advanced glycation end products (RAGE) regulates sepsis but not the adaptive immune response. J Clin Invest 2004;113:1641-50.

26. Geroldi D, Falcone C, Emanuele E. Soluble receptor for advanced glycation end products: from disease marker to potential therapeutic target. Curr Med Chem 2006;13:1971-8.

27. Parsons PE, Eisner MD, Thompson BT, et al. Lower tidal volume ventilation and plasma cytokine markers of inflammation in patients with acute lung injury. Crit Care Med 2005;33:1-6. 
28. Ranieri VM, Suter PM, Tortorella C, et al. Effect of mechanical ventilation on inflammatory mediators in patients with acute respiratory distress syndrome: a randomized controlled trial. JAMA 1999;282:54-61.

29. Eisner MD, Parsons P, Matthay MA, et al. Plasma surfactant protein levels and clinical outcomes in patients with acute lung injury. Thorax 2003;58:983-8.

30. Parsons PE, Matthay MA, Ware LB, et al. Elevated plasma levels of soluble TNF receptors are associated with morbidity and mortality in patients with acute lung injury. Am J Physiol Lung Cell Mol Physiol 2005;288:L426-31.

31. Eaton DC, Chen J, Ramosevac $\mathrm{S}$, et al. Regulation of $\mathrm{Na}$ + channels in lung alveolar type II epithelial cells. Proc Am Thorac Soc 2004;1:10-16.

32. Borok Z, Liebler JM, Lubman RL, et al. Na transport proteins are expressed by rat alveolar epithelial type I cells. Am J Physiol Lung Cell Mol Physiol 2002;282:L599-608.

33. Frank JA, Gutierrez JA, Jones KD, et al. Low tidal volume reduces epithelial and endothelial injury in acid-injured rat lungs. Am J Respir Crit Care Med 2002;165:242-9.

34. Challier M, Jacqueminet S, Benabdesselam 0, et al. Increased serum concentrations of soluble receptor for advanced glycation endproducts in patients with type 1 diabetes. Clin Chem 2005:51:1749-50.
35. Kalousova $\mathbf{M}$, Hodkova M, Kazderova $\mathbf{M}$, et al. Soluble receptor for advanced glycation end products in patients with decreased renal function. Am J Kidney Dis 2006;47:406-11.

36. Falcone C, Emanuele E, D'angelo A, et al. Plasma levels of soluble receptor for advanced glycation end products and coronary artery disease in nondiabetic men. Arterioscler Thromb Vasc Biol 2005;25:1032-7.

37. Pullerits R, Bokarewa M, Dahlberg L, et al. Decreased levels of soluble receptor for advanced glycation end products in patients with rheumatoid arthritis indicating deficient inflammatory control. Arthritis Res Ther 2005;7:R817-24.

38. Emanuele E, D'angelo A, Tomaino $C$, et al. Circulating levels of soluble receptor for advanced glycation end products in Alzheimer disease and vascular dementia. Arch Neurol 2005;62:1734-6.

39. Geroldi D, Falcone C, Emanuele E, et al. Decreased plasma levels of soluble receptor for advanced glycation end-products in patients with essential hypertension. J Hypertens 2005:23:1725-9.

40. Brookes ST, Whitely E, Egger M, et al. Subgroup analyses in randomized trials: risks of subgroup-specific analyses;: power and sample size for the interaction test. J Clin Epidemiol 2004;57:229-36.

\section{Lung alert}

\section{Six minute walk distance is a useful predictor of survival in lung transplant recipients}

Six minute walk distance (6MWD) is routinely used as part of the assessment process for lung transplant recipients. However, there is a paucity of data supporting the impact of baseline $6 \mathrm{MWD}$ on transplant outcomes in advanced lung diseases.

In this study, clinical information was prospectively collected over a 28-month period from 376 patients with diverse lung diseases awaiting lung transplantation at two centres. Investigators compared data before transplantation, after transplantation and during the overall study period, with death as the primary end point.

In each analysis every 500 foot increase in the baseline $6 \mathrm{MWD}$ was found to be a significant predictor of survival. No other variables such as age, body mass index, forced expiratory volume in $1 \mathrm{~s}$, waiting list times and native lung disease were significantly associated with survival. There was no significant interaction between the effects of $6 \mathrm{MWD}$ and the underlying lung disease.

The authors conclude that $6 \mathrm{MWD}$ is an important tool for assessing survival in lung transplant recipients for all native lung diseases. However, the study was underpowered to assess mortality in the individual lung diseases and is not generalisable to all patient populations with end stage disease. Furthermore, they do not report on any co-morbidities or physical disability which could affect $6 \mathrm{MWD}$. More research is required in assessing $6 \mathrm{MWD}$ to determine if a single baseline measurement or subsequent measurements over time offer a better prognostic tool in lung transplant candidates. The authors also suggest that rehabilitative measures that improve $6 \mathrm{MWD}$ may, in turn, affect survival following lung transplantation. This requires further study.

- Martinu T, Babyak MA, O'Connell CF, et al, for the INSPIRE investigators. Baseline 6-min walk distance predicts survival in lung transplant candidates. Am J Transplant 2008;8:1-8

\section{Ganagaram}

Correspondence to: Dr V Ganagaram, Department of Respiratory Medicine, Papworth Hospital, Cambridgeshire, UK; venkatgangaram@doctors.net.uk 